\section{Revisión sistemática de indicadores de desempeño hospitalario}

\section{Systematic review of hospital performance assessment indicators}

Melvin Morera-Salas

\section{Resumen}

Antecedentes: el objetivo de este estudio es realizar una revisión sistemática de la literatura para identificar indicadores de gestión hospitalaria con suficiente evidencia científica para que sean susceptibles de ser aplicados al grupo de hospitales de la Caja Costarricense de Seguro Social.

Métodos: se identificaron los estudios relevantes publicados entre el $1^{\circ}$ de marzo de 2000 y el 30 de setiembre de 2011 mediante una búsqueda en MEDLINE. Se utilizaron los siguientes descriptores: "hospital performance indicators", "hospital inpatient quality indicators", "hospital-performance y top 100 hospitals".

Resultados: de los 994 artículos identificados, 56 cumplieron los criterios de inclusión: 5\% mide el impacto de las estrategias de calidad en los resultados finales del hospital, 13\% son revisiones sistemáticas sobre indicadores de desempeño hospitalario, $22 \%$ describe el marco conceptual y los indicadores de proyectos de calidad y $60 \%$ incluye temas metodológicos varios sobre indicadores de gestión.

Se identificaron 23 agencias que miden el desempeño hospitalario. Asociados a estas agencias existen 383 indicadores. El 51\% de estos indicadores son de seguridad al paciente, 29\% de efectividad y $10 \%$ relacionadas con la oportunidad y la atención centrada en el paciente.

Conclusión: existen un amplio grupo de indicadores de desempeño hospitalario que son susceptibles de ser aplicados en el sistema hospitalario público de Costa Rica. Para lograr su implementación se deben cumplir con una serie de requisitos: revisión del marco de calidad existente actualmente, definición de las medidas, recopilación de datos, análisis e interpretación apropiados y las actuaciones sobre los resultados.

Descriptores: Calidad de la Atención de Salud, Indicadores de Calidad de la Atención de Salud, Garantía de la Calidad de Atención de Salud (FUENTE: DeCS).

\section{Abstract}

Background: The objective of this study is to undertake a systematic review of the international scientific literature to identify hospital performance indicators which have the potential to be implemented in the Costa Rican public hospital system.

Methods: A literature review was undertaken using MEDLINE to identify relevant studies published between March 1, 2000 and September 30, 2011. The following search terms were used: "hospital performance indicators", "hospital inpatient quality indicators", "hospital performance and top 100 hospitals".
Afiliación del autor: Dirección Compra de Servicios de Salud, Caja Costarricense de Seguro Social

凶mmoreras@ccss.sa.cr 


\section{Indicadores de desempeño hospitalario / Morera-Salas}

Results: From 994 potentially relevant articles, 56 met the inclusion criteria: 5\% measure the impact of quality-strategies on hospital final outputs, $13 \%$ are systematic reviews about hospital performance indicators, $22 \%$ describe the conceptual framework and the indicators for quality projects and $60 \%$ include various methodological topics about hospital performance indicators.

We identified 23 agencies that measure hospital performance. There are 383 indicators related to these agencies: $51 \%$ are indicators on patient safety, $29 \%$ on efficiency and $10 \%$ are related to the timeliness and patient-centered care.

Conclusion: There is large number of available hospital performance indicators that could be applied in the Costa Rican public hospital system. In order to be successfully implemented, they must meet certain requirements: review of the current quality framework, definition of the measures, data collection, adequate analysis and interpretation and action upon the results.

Key words: Quality of healthcare, quality of healthcare indicators, quality assurance in healthcare. (source: MESH).

Fecha recibido: 17 de diciembre de 2012

Fecha aceptado: 07 de febrero de 2013

La calidad de la atención hospitalaria ha tenido un rápido crecimiento en los últimos años. Este campo de investigación surge como un tema innovador hace aproximadamente 15 años y actualmente hay muchos proyectos de este tipo funcionando en todo el mundo. Este proceso se ha visto influenciado en parte porque varias iniciativas están siendo apoyadas por organizaciones internacionales como la Organización Mundial de la Salud (OMS) y la Organización para la Cooperación y el Desarrollo Económicos (OCDE).

Otro factor que ha influido en el desarrollo de indicadores de desempeño hospitalario es la demanda de informes de evaluación de resultados asociados a la rendición de cuentas de muchos sistemas de salud.

El gran número de publicaciones existentes en el campo de la gestión hospitalaria, dificulta que los profesionales encargados de la evaluación y gestión de servicios de salud se mantengan adecuadamente informados. Por este motivo, las revisiones sistemáticas de la literatura científica se han vuelto herramientas esenciales para estar adecuadamente informados y facilitar el proceso de toma de decisiones informadas.

La revisión sistemática es una forma de investigación que proporciona un resumen de los estudios existentes sobre un determinado tema, utilizando para ello métodos explícitos y sistemáticos de identificación, evaluación crítica y síntesis de la literatura científica. ${ }^{1}$

Dado que las revisiones sistemáticas son un ejercicio científico, e influye en las decisiones que afecta a la gestión y administración de los cuidados en salud, debe ser tan rigurosa como cualquier otra investigación. ${ }^{2}$

La calidad de una revisión y por ende su valor, depende de si utiliza métodos de revisión científicos para minimizar el riesgo de error y los sesgos. ${ }^{3,4}$ Lo que diferencia a las revisiones sistemáticas de las revisiones tradicionales de la literatura es el uso de estos métodos explícitos y rigurosos. En este sentido las revisiones sistemáticas utilizan métodos formales y objetivos para seleccionar e interpretar los estudios y por ente, se evita el sesgo en los resultados y las conclusiones.

Una parte fundamental de toda revisión bibliográfica consiste en localizar las investigaciones realizadas sobre el tema de interés. El objetivo del proceso de revisión sistemática es la evaluación de la validez de todos los estudios identificados en la búsqueda. La razón por la que se realiza esta valoración es que, al excluir los estudios de menor calidad, se reduce tanto el riesgo de error como los sesgos introducidos en los resultados de la revisión. ${ }^{5}$

Elobjetivo de este estudio es realizar una revisión sistemática en MedLine para identificar indicadores de desempeño o gestión hospitalaria con suficiente evidencia científica para ser aplicados en los hospitales de la Caja Costarricense de Seguro Social.

\section{Métodos}

\section{Bases de datos a estudiar}

Se efectuó una búsqueda en MEDLINE desde el $1^{\circ}$ de marzo de 2000 hasta el 30 de setiembre de 2011. También se realizó una búsqueda en citas bibliográficas y las listas de referencia de todos los artículos encontrados sobre el tema de interés, para aumentar la posibilidad de identificar todos los estudios relevantes, las cuales se identificaron mediante el motor de búsqueda Google académico.

\section{Estrategia de búsqueda}

Se utilizaron los siguientes términos de búsqueda: hospital performance indicators, hospital inpatient quality indicators, hospital-performance y top 100 hospitals.

La búsqueda se hace por título, resumen o palabras clave. Para el filtro también se programó para que se contabilizaran sólo los artículos que tengan acceso libre con lo que se puedan revisar detalles metodológicos del cálculo de los indicadores. 
Cuadro 1. Grupo de indicadores y sus direcciones Web

\section{Organización}

Estados Unidos

Agency for Healthcare Research and Quality (AHRQ)

-Indicadores de Seguridad del Paciente

-Indicadores de calidad para pacientes

hospitalizados

-Indicadores pediátricos

National Quality Forum (NQF)

Joint Commission on the Accreditation of Healthcare Organizations (JCAHO)

Centers for Medicaid and Medicare Services (CMMS)/Hospital Quality Alliance (HQA)

Leapfrog Hospital Quality and Safety Survey

Centre for Disease Control (CDC)

- Public Reporting of Healthcare

- Associated Infections: A toolkit

\section{Canadá}

Canadian Institute of Health Performance (CIHI)

Reino Unido

Healthcare Commission

National Centre for Health Outcome Development Indicators (NCHOD)

España

lasist

-Indicadores de la gestión global del hospital

- Seis áreas específicas: Sistema Nervioso

Respiratorio, Corazón, Cirugía Digestiva,

Traumatología y Ortopedia, y Área de

la Mujer

Europa

European Commission: Safety

Improvement for Patients in Europe

(SImPatlE)

National Indicator Project, Denmark (NIP) http://www.qualityforum.org/pdf/reports/sre/txsreexecsummarypublic.pdf

http://www.qualityforum.org/publications/reports/hospital_measures.asp 


\section{Indicadores de desempeño hospitalario / Morera-Salas}

\begin{tabular}{|c|c|c|}
\hline $\begin{array}{l}\text { The Good Medical Department, } \\
\text { Denmark (DGMA) }\end{array}$ & http://www.dgma.dk/ (not current) & 10 \\
\hline $\begin{array}{l}\text { Performance Indicators on Patient Safety } \\
\text { and Effectiveness for Dutch Hospitals }\end{array}$ & http://www.medscape.com/viewarticle/574641_8 & 26 \\
\hline \multicolumn{3}{|l|}{ Australia } \\
\hline $\begin{array}{l}\text { Australian Institute of Health and } \\
\text { Welfare (AlHW) }\end{array}$ & http://www.aihw.gov.au/publication-detail/?id = $10737418683 \varepsilon t a b=2$ & 14 \\
\hline $\begin{array}{l}\text {-Reporte nacional sobre desempeño del } \\
\text { sector salud }\end{array}$ & http://www.aihw.gov.au/indicators/index.cfm & \\
\hline \multicolumn{3}{|l|}{-Reporte de eventos centinela } \\
\hline $\begin{array}{l}\text { Australian Council of Healthcare } \\
\text { Standards (ACHS) }\end{array}$ & http://www.achs.org.au/Clinicallndicators/ & 152 \\
\hline $\begin{array}{l}\text { Queensland Health Measured Quality } \\
\text { Hospital Report }\end{array}$ & http://www.health.qld.gov.au/quality/mq_reports2004.asp & 27 \\
\hline $\begin{array}{l}\text { NSW Therapeutic Advisory Group; } \\
\text { Medication Safety - PIMS }\end{array}$ & http://www.ciap.health.nsw.gov.au/nswtag/pims.html & 27 \\
\hline $\begin{array}{l}\text { Key Performance Indicators for Australian } \\
\text { Public Mental Health Services }\end{array}$ & http://www.mhnocc.org/Benchmarking/KPIDevelopment/ & 6 \\
\hline $\begin{array}{l}\text { Department of Human Services Victoria, } \\
\text { Maternity Services }\end{array}$ & http://www.health.vic.gov.au/maternitycare & 11 \\
\hline \multicolumn{3}{|l|}{-Indicadores de desempeño } \\
\hline \multicolumn{3}{|l|}{$\underline{\text { Internacionales }}$} \\
\hline $\begin{array}{l}\text { International Quality Indicator Project } \\
\text { (IQIP) }\end{array}$ & http://www.internationalqip.com/ & 47 \\
\hline $\begin{array}{l}\text { World Health Organization, Performance } \\
\text { Assessment Tool for QI in Hospitals } \\
\text { (PATH) }\end{array}$ & $\begin{array}{l}\text { http://www.pathqualityproject.eu/what_is_path.html } \\
\text { http://www.pathqualityproject.eu/upLoad/file/path_20092010_indicators_ } \\
\text { descriptive_sheets.pdf }\end{array}$ & 25 \\
\hline $\begin{array}{l}\text { Organization for Economic Co-operation } \\
\text { and Development (OECD) }\end{array}$ & $\begin{array}{l}\text { http://www.oecd.org/document/11/0,3343, } \\
\text { en_2649_33929_16502667_1_1_1_1,00.html }\end{array}$ & 49 \\
\hline -Indicadores a nivel del sistema de salud & $\begin{array}{l}\text { http://www.oecd.org/findDocument/0,3770, } \\
\text { en_2649_33929_1_119656_1_1_1,00.html }\end{array}$ & \\
\hline $\begin{array}{l}\text {-Indicadores de calidad de la atención } \\
\text { de salud a nivel de los sistemas de salud }\end{array}$ & http://www.oecd.org/dataoecd/28/32/33865630.pdf & \\
\hline $\begin{array}{l}\text {-Calidad de la atención de la salud } \\
\text { mental a nivel de los sistemas de salud } \\
\text { en los países de la OCDE }\end{array}$ & http://www.oecd.org/dataoecd/28/35/33865450.pdf & \\
\hline -Indicadores de atención cardiaca & & \\
\hline
\end{tabular}

Finalmente, los estudios duplicados dentro o entre grupo de palabras clave fueron eliminados.

\section{Criterios de inclusión}

Los criterios de inclusión están basados en la pregunta de revisión "¿Cuáles estudios cuentan con una descripción metodológica sobre el cálculo de indicadores de desempeño o gestión hospitalaria?”. Este criterio de inclusión permitirá reflejar explícitamente el enfoque, la naturaleza y los límites de la revisión.

También se incluye como el criterio de selección adicional que deben ser estudios que se realizan para medir el desempeño hospitalario.

Se revisaron de forma independiente los artículos y se comprobó el cumplimiento de los criterios de inclusión. 
Extracción de datos y evaluación de la calidad

Se evaluó independientemente la calidad metodológica de los estudios y se realizó una extracción de datos (indicadores de desempeño y forma de cálculo), utilizando una hoja de recogida de datos diseñada previamente.

\section{Resultados}

Como resultado de la búsqueda se identificaron 994 estudios. De estos 850 fueron excluidos tras la revisión del título y el resumen, pues no hacían referencia a indicadores de gestión hospitalaria. Treinta y seis nuevos artículos fueron obtenidos tras la revisión de las referencias bibliográficas de los estudios. A 144 artículos se les revisó la metodología y resultados, de los cuales se descartaron 124 por no incluir la ficha técnica de los indicadores. Finalmente 56 estudios se revisaron y se clasificaron para estudiar en detalle el marco metodológico y las fichas técnicas de los indicadores de desempeño hospitalario.

De los 56 artículos revisados un 39\% está basado en la aplicación de algunos indicadores a entornos específicos, principalmente a hospitales de Estados Unidos, Europa y Australia. ${ }^{6-27}$ Un $22 \%$ de las investigaciones se refieren al detalle del marco teórico o los indicadores de las principales agencias de gestión hospitalaria , ${ }^{28-39}$ un $21 \%$ son aplicaciones metodológicas de ajustes por riesgo y análisis de indicadores específicos. ${ }^{40-50} \mathrm{El} 13 \%$ corresponden a revisiones sistemáticas de la literatura y de éstos tres están basados en una revisión detallada de artículos pertenecientes a agencias de evaluación del desempeño hospitalario. ${ }^{51-57}$ El restante $5 \%$ se relaciona la asociación entre la aplicación de las estrategias de mejora de la calidad en los hospitales y el éxito de los hospitales en el cumplimiento de los requisitos de calidad definidos que se consideran productos intermedios del proceso de atención. ${ }^{58-60}$

\begin{tabular}{|c|c|c|c|c|c|c|}
\hline \multicolumn{7}{|c|}{$\begin{array}{c}\text { Cuadro 2. Dimensiones de calidad para elaboración de indicadores de desempeño } \\
\text { hospitalario según agencias seleccionadas }\end{array}$} \\
\hline Dimensiones & uK & Canada & $\begin{array}{l}\text { Australia- } \\
\text { ACHS }\end{array}$ & $\begin{array}{l}\text { USA- } \\
\text { AHRQ }\end{array}$ & $\begin{array}{l}\text { WHO- } \\
\text { PATH }\end{array}$ & $\begin{array}{l}\text { OECD- } \\
\text { HCQI }\end{array}$ \\
\hline Aceptabilidad & & $\checkmark$ & & & & \\
\hline Accesibilidad & $\checkmark$ & $\checkmark$ & $\checkmark$ & $\checkmark$ & & $\checkmark$ \\
\hline Adecuación & & $\checkmark$ & $\checkmark$ & & & \\
\hline Cuidado del medio ambiente y servicios & $\checkmark$ & & & & & \\
\hline Competencia & & $\checkmark$ & & & & \\
\hline Capacidad & & & $\checkmark$ & & & \\
\hline Continuidad & & $\checkmark$ & $\checkmark$ & & & \\
\hline Eficacia & $\checkmark$ & $\checkmark$ & $\checkmark$ & $\checkmark$ & & $\checkmark$ \\
\hline Mejorar la salud & & & & & $\checkmark$ & \\
\hline Enfoque clínico, gastos o costos & & & & & $\checkmark$ & $\checkmark$ \\
\hline Eficiencia & & $\checkmark$ & $\checkmark$ & $\checkmark$ & $\checkmark$ & $\checkmark$ \\
\hline Equidad & $\checkmark$ & $\checkmark$ & $\checkmark$ & $\checkmark$ & $\checkmark$ & $\checkmark$ \\
\hline Gobernabilidad & $\checkmark$ & & & & & \\
\hline Atención centrada en el paciente & $\checkmark$ & $\checkmark$ & $\checkmark$ & $\checkmark$ & $\checkmark$ & $\checkmark$ \\
\hline Seguridad & $\checkmark$ & $\checkmark$ & $\checkmark$ & $\checkmark$ & & \\
\hline Sostenibilidad & & & $\checkmark$ & & & \\
\hline Oportunidad & $\checkmark$ & & & $\checkmark$ & & \\
\hline
\end{tabular}


Cuadro 3. Dimensiones e indicadores incluidos en el "Top 20 Hospitales" de la agencia de evaluación lasist

\begin{tabular}{|c|c|}
\hline Dimensión & Indicador \\
\hline Calidad & $\begin{array}{l}\text { Índice de mortalidad ajustada por riesgo } \\
\text { Índice de complicaciones ajustada por riesgo } \\
\text { Índice de readmisiones ajustada por riesgo }\end{array}$ \\
\hline Adecuación de la práctica clínica & $\begin{array}{l}\text { Índice de sustitución de cirugías sin ingreso } \\
\text { Índice de hospitalizaciones evitables ajustado }\end{array}$ \\
\hline Eficiencia & $\begin{array}{l}\text { Índice de estancias ajustado por riesgo } \\
\text { Productividad (unidades de producción/trabajador) } \\
\text { Coste de aprovisionamiento por unidad de producción ajustada }\end{array}$ \\
\hline
\end{tabular}

\section{Agencias de evolución del desempeño hospitalario}

Se analizaron en detalle dos de los artículos de revisiones sistemáticas que estudian los indicadores implementados por las agencias de gestión hospitalaria. Se identifica 23 organizaciones o agencias de evaluación de desempeño hospitalario en Estados Unidos, Canadá, Inglaterra, Australia, varios países europeos y países asociados a la OCDE. ${ }^{53}$

En el cuadro 1, se incluye el nombre de las principales Agencias, su país de origen, el número de indicadores que componen cada lista y la dirección web donde se pueden ver los detalles tanto de la lista de los indicadores de desempeño como el marco teórico que les da sustento.

\section{Marco teórico para el desarrollo indicadores hospitalarios según agencia de evaluación internacionales}

Los primeros indicadores de desempeño hospitalario utilizaban como marco conceptual la taxonomía dada por Donabedian, ${ }^{51}$ quien clasifica las medidas si están relacionadas con la estructura, proceso o el resultado. Actualmente las principales agencias de evaluación han desarrollo un marco de calidad que sustenta sus indicadores. En el cuadro 2, se muestra el detalle de las dimensiones para un grupo seleccionado de agencias de evaluación de desempeño hospitalario, donde se destacan los tópicos de accesibilidad, eficacia, eficiencia, equidad, atención centrada en el paciente y seguridad.

\section{Tipo de indicadores de gestión hospitalaria según agencias de evaluación internacionales}

Las agencias de evaluación del desempeño hospitalario poseen 383 indicadores de los cuales muchos son comunes entre ellas. Agrupando los indicadores se tiene que 104 son generales, 100 son quirúrgicos y 179 del área no quirúrgica. Un $39 \%$ de los indicadores son de resultados, $7 \%$ de estructura y $54 \%$ de proceso. ${ }^{53}$

Si se utiliza para su clasificación el marco conceptual de la Herramienta de Evaluación de Desempeño para el Mejoramiento de la Calidad en Hospitales de la Organización
Mundial de la Salud (PATH por sus siglas en inglés) se observa que 51\% de los indicadores están relacionados con la dimensión de seguridad (paciente, trabajadores y al ambiente), un $29 \%$ se clasifican en la dimensión de efectividad, 10\% para cada una de las dimensiones relacionadas con la oportunidad y la atención centrada en el paciente. ${ }^{53}$

A nivel países de habla hispana el más destacado grupo de indicadores es el realizado por la empresa española Iasist y que se denomina "Top 20 Hospitales". La muestra incluye hospitales públicos y privados que voluntariamente participan aportando la información de base para su cálculo. El índice está compuesto por ocho indicadores recogidos en las dimensiones de calidad, adecuación de la práctica clínica y eficiencia ${ }^{38}$. En el cuadro 3, se muestra el título de los indicadores de cada una de estas dimensiones.

Adicionalmente estos y otros indicadores se aplican para un grupo de especialidad médicas: sistema nervioso, área de respiratorio, corazón, cirugía digestiva, traumatología y ortopedia y área de la mujer. ${ }^{38}$

\section{Discusión}

Con la revisión sistemática de la literatura científica se identificaron 56 estudios que cumplieron los criterios de inclusión los cuales se revisaron y se clasificaron, para estudiar en detalle el marco metodológico y las fichas técnicas de los indicadores de desempeño hospitalario.

Tras la revisión de los estudios se identifican 20 agencias nacionales y tres internacionales que implementan indicadores de gestión hospitalaria. La revisión detallada de los grupos de indicadores de las principales agencias da cuenta de 383 indicadores, de los cuales muchos son comunes entre ellas.

Dentro de las principales limitaciones de la utilización de sistemas de indicadores para la evaluación de la gestión hospitalaria están: sobre interpretación de algunos indicadores que puede llevar a juicios sobre la calidad subyacente de la atención; riesgo a la estigmatización a una institución y provocar enormes tensiones y desviar la atención hacia una mejora 
superficial vía manipulación del sistema; los datos comparativos de resultados pueden ser utilizados por agentes externos para hacer juicios sobre la calidad de la atención hospitalaria; los indicadores de gestión tienen menor sensibilidad para identificar problemas de calidad de la atención en las unidades de atención de agudos respecto de las quirúrgicas de alto riesgo.

Un tema para investigaciones futuras es la revisión detallada de los marcos teóricos de las principales agencias de evaluación y compararlas con la del Compromiso de Gestión de la Caja Costarricense de Seguro Social (CCSS) actual y ver la conveniencia de posibles modificaciones.

Otra línea de investigación que se recomienda es analizar los indicadores de las principales agencias, así como los requerimientos de información y organización para su implementación en hospitales de la CCSS.

Se recomienda efectuar las investigaciones necesarias para ajustar ciertos indicadores de acuerdo a las condiciones del paciente (complejidad y comorbilidad) ${ }^{42-44}$ y características de los hospitales, de tal forma que permitan ser utilizados en comparaciones en el espacio y el tiempo y dentro y entre hospitales o servicios hospitalarios.

Otro tema que se debería considerar para investigaciones futuras es analizar si la implementación de un sistema de indicadores de gestión, tipo Compromisos de Gestión, está relacionada con un aumento en la calidad de la atención. La conclusión básica de las investigaciones es que no es suficiente con aplicar un sistema de indicadores y evaluarlos, hay que hacer devoluciones de resultados e implementar otras estrategias de educación a los hospitales. ${ }^{57,60}$

Los resultados de algunos estudios indican que la aplicación de estrategias internas y externas de mejora de la calidad en los hospitales tiene efectos beneficiosos en los productos estudiados del hospital. ${ }^{57,59}$ Un estudio realizado para países de la Unión Europea demostró asociaciones positivas seis estrategias internas de mejora de la calidad y resultados intermedios del hospital, explicando aproximadamente la mitad de su variación. ${ }^{14}$

Como conclusión se tiene que no obstante las limitaciones mencionadas anteriormente, los datos de resultados de evaluación de indicadores de desempeño son útiles para investigaciones de tendencias y monitoreo dentro de un hospital. ${ }^{61}$ En este sentido la OMS recomienda utilizar los indicadores de desempeño para:

a. Dar orientaciones para la acción de los directores y profesionales en general de los hospitales.

b. Contribuir a la mejora de los sistemas de información y la calidad de los datos.

c. Reforzar la credibilidad de los resultados de sistemas de medición y la confianza de los hospitales en los datos que necesitan para evaluar.

d. Promover la rendición de cuentas.

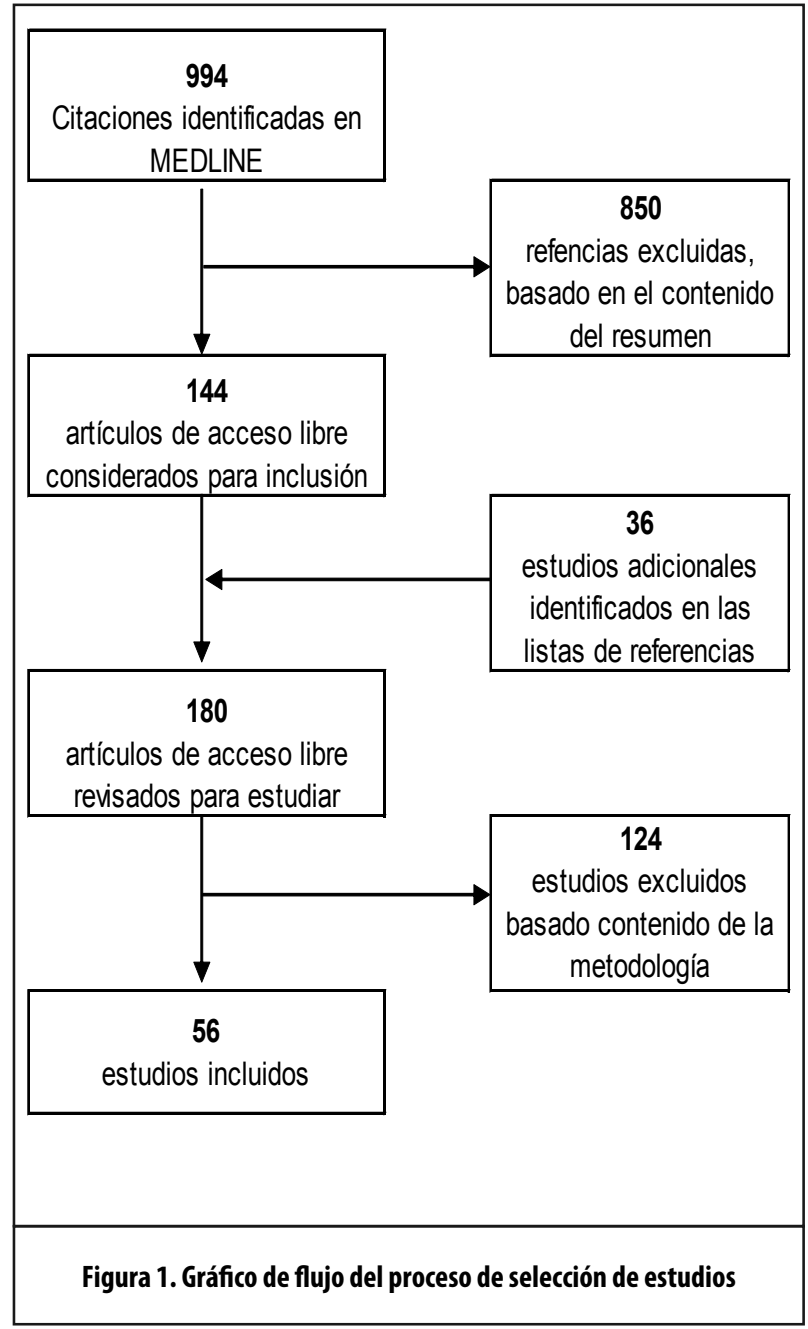

La futura implementación de un grupo de indicadores de desempeño hospitalaria debe poseer un rigor científico, empezando por el desarrollo de un marco de calidad, seguido por cuatro etapas posteriores para su aplicación: definición de las medidas; recopilación de datos; análisis e interpretación apropiados de resultados; actuaciones sobre los resultados.

Es importante mencionar también, que los informes de gestión hospitalaria deben ser emitidos y publicados por cada hospital de una manera uniforme, de modo que permita la comparación entre centros y sea un punto de partida para que los interesados puedan comenzar a hacer preguntas en cuanto a la gestión y la eficiencia.

Conflicto de interés: no existe conflicto de interés.

\section{Referencias}

1. Pai M, McCulloch M, Gorman JD, Pai N, Enanoria W, Kennedy $G$ et al. Systematic reviews and meta analyses: an illustrated, step-by-step guide. Natl Med J India 2004; 17:86-95. 


\section{Indicadores de desempeño hospitalario / Morera-Salas}

2. Sackett D, Strauss S, Richardson W, Rosenberg W, Haynes R. Evidence-based medicine: how to practice and teach EBM. 2 nd. Ed. London: Churchill-Livingstone; 2000.

3. Centro Colaborador Español del Instituto Joanna Brigss Institute para los Cuidados de Salud Basados en la Evidencia. Introducción a las revisiones sistemáticas. Reproducido del Best Practice 2001;Sup 1:1-6

4. The Cochrane Collaboration. The Cochrane Manual Issue 1, 2007.

5. Valoración de las revisiones sistemáticas. Best Practice 2000; 1:1-6.

6. Courtney E, Ankrett S, McCollum P. 28-Day emergency surgical re-admission rates as a clinical indicator of performance. Ann $R$ Coll Surg Engl 2003; 85:75-8.

7. Lutfiyya N, Bhat Deepa, Gandhi S, Nguyen C, Weidenbacher V, Lipsky M. A comparison of quality of care indicators in urban acute care hospitals and rural critical access hospitals in the United States. Int J Qual Health Care; 19:141-9.

8. Solon O, Woo K, Quimbo S, Shimkhada R, Florentino J, Peabody J. A novel method for measuring health care system performanceexperience from OIDS in the Philippines. Health Policy Plan 2009; 24:167-74.

9. Lombarts M, Rupp I, Vallejo P, Suñol R, Klazinga N. Application of quality improvement strategies in 389 European hospitals- results of the MARQuIS project. Qual Saf Health Care 2009; 18:i28-37.

10. Rivard P, Elwy A R, Loveland S, Zhao S, Tsilimingras D, Elixhauser A, Romano P S, Rosen A K. Applying Patient Safety Indicators (PSIs) across Healthcare Systems: Achieving Data Comparability. In: Henriksen K, Battles J B, Marks E, Lewin D I, editors. Advances in Patient Safety: From Research to Implementation. Rockville, MD: Agency for Healthcare Research and Quality; 2005; 2:7-25.

11. Jha Ashish, Li Z, Orav J Epstein A. Care in US hospital- The Hospital Quality Alliance program. N Engl J Med 2005; 353:265-74.

12. Kerssens J, Groenewegen P, Sixma H, Boerma W, van der Eijk I. Comparison of patient evaluations of health care quality in relation to WHO measures of achievement in 12 European countries. Bull World Health Organ 2004; 82:106-14.

13. Peters R. Hospital performance indicators in the netherland-a preterm delivery. Neth Heart J 2009; 17: 48-9.

14. Marshall MN, Romano PS. Impact of reporting hospital performance. Qual Saf Health Care 2005; 14:77-8.

15. DesHarnais S, McMahon L, Wroblewski R. Measuring Outcomes of Hospital Care Using Multiple Risk-Adjusted Indexes. Health Serv Res 1991; 26:425-45.

16. Ranking 35 hospitales y clínicas de América Latina. Disponible en: http://rankings.americaeconomia.com/2010/clinicas/ metodologia. Consultado 10 de Noviembre de 2011.

17. Gobierno Federal de México. Observatorio del Desempeño Hospitalario 2009. Secretaría de Salud. $1^{\circ}$ Ed. 2010. México D.F.

18. Miller M, Elixhauser A, Zhan Ch, Meyer G. Patient Safety Indicators - using administrative data to identify potential patient safety concerns. Health Serv Res 2001; 36:110-32.

19. Jian W, Huang Y, Hu M, Zhang X. Performance evaluation of inpatient service in Beijing- a horizontal comparison with risk adjustment based on Diagnosis Related Groups. BMC Health Serv Res 2009; 9:72.

20. Gibberd R. Performance measurement- is it now more scientific. Int J Qual Health Care 2005; 17:185-6.
21. Chen J, Radford M, Wang Y, Marciniak T, Krumholz H. Performance of the "100 top hospitals": what does the report card report? Health Aff 1999; 18:53-68.

22. Scott I, Ward M. Public reporting of hospital outcomes based on administrative data- risks and opportunities. Med J Aust 2006; 184:571-5.

23. Bardach N, Chien A, Dudley A. Small Numbers Limit the Use of the Inpatient Pediatric Quality Indicadors for hospital comparison. Acad Pediatr. 2010; 10:266-73.

24. Hines A, Stranges E, Andrews RM. Trends in Hospital RiskAdjusted Mortality for Select Diagnoses by Patient Subgroups, 2000-2007: Statistical Brief \#98. 2010 Oct. En: Healthcare Cost and Utilization Project (HCUP) Statistical Briefs [Internet]. Rockville (MD): Agency for Health Care Policy and Research (US); 2006 Feb. Disponible en: http://www.ncbi.nlm.nih.gov/ books/NBK52657/

25. Romano P, Mull H, Rivard P, Zhao Sh, Henderson W, Loveland S, Tsilimingras D, Christiansen C, Rosen A. Validity of selected AHRQ patient safety indicators based on VA National Surgical Quality Improvement Program data. Health Serv Res 2009; 44:182-204.

26. Shahian D, Wolf R, Iezzoni L, Kirle L, Normand S. Variability in the measurement of hospital-wide mortality rates. N Engl J Med 2010; 363:2530-9.

27. Groene O, Klazinga N, Kazandjian V, Lombrail P, Bartels P. WHO Performance Assessment Tool for Quality Improvement in Hospitals PATH- an analysis of the pilot implementation in 37 hospitals. Int J Qual Health Care 2008; 20:155-61.

28. Thomson Reuters. 100 Top hospitals: National Benchmarks 2009 Study. Thomson Reuters $17^{\text {th }}$ ed. march 2010.

29. Arah OA, Westert G, Hurst J, Klazinga NS. A conceptual framework for the OECD Health Care Quality Indicators project. Int J Qual Health Care 2006; 18:5-13.

30. Veillard J, Champagne F, Klazinga N, Kazandjian V, Arah OA, Guisset al. A performance assessment framework for hospitals: the WHO regional office for Europe PATH project. Int J Qual Health Care 2005; 17:487-96.

31. Australasian Clinical Indicator Report: 2001 - 2008. Determining the Potential to Improve Quality of Care: 10th Edition Australian Council on Healthcare Standards, 2008.

32. Collopy B. Clinical indicators in accreditation- an effective stimulus to improve patient care. Int J Qual Health Care 2000; 12: 211-16.

33. Garcia S, Gil ML, Wei L, Kelley E, the Members of the HCOI Expert Group. Health Care Quality Indicators Project 2006 Data Collection Update Report. OECD Health Working Papers 29, 2007.

34. García-Eroles L, Illa C, Arias A, Casas M. Los Top 20 2000: objetivos, ventajas y limitaciones del método. Rev Calidad Asistencial 2001; 16:107-16.

35. US Department of Health and Human Services, Agency for Healthcare Research and Quality. National Healthcare Quality Report 2010. Rockville, MD: Agency for Healthcare Research and Quality, 2011.

36. World Health Organization. Performance Assessment Tool for Quality Improvement in Hospitals: Indicators descriptive sheets 09/10. Division of Country Health Systems, WHO Regional Office for Europe, 2010. 
37. World Health Organization. Performance Assessment Tool for Quality Improvement in Hospitals. WHO Regional Office for Europe, 2007.

38. Iasist. Hospitales Top 20 Benchmarks para la excelencia 2011. 1 $1^{a}$ Ed. Iasist, S.A. España, 2011. URL: http://www.iasist.com/files/ TOP_20_2011_publicacion.pdf. Consultado 10 de noviembre 2011.

39. Thomson R, Taber S, Lally J, Kazandjian V. UK Quality Indicator Project (UK OIP) and the UK independent health care sector: a new development. Int J Qual Health Care 2004; 16:151-6.

40. World Health Organization. A review of determinants of hospital performance. WHO, District Health Systems, Geneva 1994.

41. Austin P. Bayes rules for optimally using bayesian hierarchical regression models in provider profiling to identify high-mortality hospitals. BMC Med Res Methodol 2008; 8:30.

42. Shahian D, Normand Sh. Comparison of risk-adjusted hospital outcomes. Circulation 2008; 117:1955-63.

43. Li P, Kim M, Doshi J. Comparison of the performance of Elixhauser comorbidity measures in predicting mortality. BMC Health Serv Res 2010; 10:245.

44. Li B, Evans D, Faris P, Dean S, Quan H. Risk adjustment performance of Charlson and Elixhauser comorbidities in ICD-9 and ICD-10 administrative databases. BMC Health Serv Res 2008, 8:12.

45. Stausberg J, Halim A, Färber R. Concordance and robustness of quality indicator sets for hospitals-an analysis of routine data. Health Serv Res 2011; 11:106.

46. Noyez L. Control charts, Cusum techniques and funnel plots. A review of methods for monitoring performance in healthcare. Int Cardio Vasc Thorac Surg 2009; 9:494-9.

47. Van Lent W, de Beer R, Van Harten W. International benchmarking of specialty hospitals. A series of case studies on comprehensive cancer centres. BMC Health Serv Res 2010; 10:253.

48. Hannan E, Magaziner J, Wang J, Eastwood E, Silberzweig S, Gilbert M, Morrison S, McLaughlin MA, Orosz G, Siu A. Mortality and locomotion 6 months after hospitalization for hip fracture- risk factors and risk-adjusted hospital outcomes. JAMA 2001; 285(21):2736-42.

49. Silveira A, Machado M, Riboldi J, Guimarães J. Mortality prediction model using data from the Hospital Information System. Rev. Saúde Públ. 2010; 44:1-8.
50. Donabedian A. Twenty years of research on the quality of medical care. Eval. Health Prof. 1985; 8:243-65.

51. Giordani F, Rozenfeld S. Adverse drug events in hospitals- a systematic review. Cad. Saúde Pública Rio de Janeiro 2009; 25:S360-72.

52. Groene O, Skau JK, Frolich A. An international review of projects on hospital performance assessment. Int J Qual Health Care 2008; 20:162-71.

53. Copnell B, Hagger V, Wilson SG, Evans SM, Sprivulis PC, Cameron PA. Measuring the quality of hospital care: an inventory of indicators. Intern Med J. 2009; 39:352-60.

54. Haller G, Stoelwinder J, Myles P, McNeil J. Quality and Safety Indicators in Anesthesia: A Systematic Review. Anesthesiology 2009; 110:1158-75.

55. Davies S, Geppert J, McClellan M, McDonald K, Romano P, Shojania K. Refinement of the HCUP Quality Indicators. Agency for Healthcare Research and Quality; USA, May 2001.

56. Ross J, Mulvey G, Stauffer B, Patlolla V, Bernheim S, Keenan P, Krumholz H. Statistical models and patient predictors of readmission for heart failure: a systematic review. Arch Intern Med 2008; 168:1371-86.

57. De Vos M, Graafmans W, Kooistra M, Meijboom B, Van Der Vort P, Westert G. Using quality indicators to improve hospital care: a review of the literature. Int J Qual Health Care 2009; 21:119-29.

58. López L, Hicks L, Cohen A, McKean S, Weissman J. Hospitalists and the Quality of Care in Hospitals. Arch Intern Med 2009; 169:1389-94.

59. Suñol R, Vallejo P, Thompson A, Lombarts M J M H, Shaw C D, Klazinga N. Impact of quality strategies on hospital outputs. Qual Saf Health Care 2009; 18:162-8.

60. Gibberd R, Hancock S, Howley P, Kay R. Using indicators to quantify the potential to improve the quality of health care. Int J Qual Health Care 2004; 16: i37-43.

61. Lilford R, Mohammed MA, Spiegelhalter D, Thomson R: Use and misuse of process and outcome data in managing performance of acute medical care: avoiding institutional stigma. Lancet 2004, 363:1147-54. 